\section{Comparative effectiveness of combined oral contraceptives in adolescents}

Since the recent publication of our study on the comparative effectiveness of combined oral contraceptives (COCs) in relation to progestogen type, length of pill-free interval and body mass index (BMI) during typical clinical use, ${ }^{1}$ we have received requests for further information. Specifically, we were asked if adolescents, who are known to have higher missed pill rates and thus higher unintended pregnancy rates, specifically benefit from shortening the pill-free interval. In our study this specifically refers to a shortening of the pill free interval to 4 days compared to the conventional 7-day intake break.

First, we would like to summarise the main study results then provide specific data for adolescents. In the International Active Surveillance of Women Taking Oral Contraceptives (INAS) study, contraceptive effectiveness was investigated in a large (52 218 US participants), prospective, controlled, non-interventional, long-term cohort study with active surveillance. Loss to follow-up was low and contraceptive failure rates were described by both Pearl Index (PI) and life-table analysis. Inferential statistics for contraceptive failure were based on Cox regression models.

Based on 1634 unintended pregnancies during 73269 woman-years of COC exposure, we found that COCs that contained progestogens with longer halflives (e.g. drospirenone) in combination with 24-day intake regimens appeared to provide better contraceptive effectiveness compared to COCs taken in the conventional 21-day regimen. Lifetable estimates of contraceptive failure for a 24-day regimen of drospirenone and ethinylestradiol and 21-day regimens of other progestogens were $2.1 \%$ and $3.5 \%$ after the first study year, and $4.7 \%$ and $6.7 \%$ after the third year. The hazard ratio (HR) adjusted for age, BMI, parity and educational level was 0.7 (95\% CI 0.6-0.8). Furthermore, the direct comparisons of the 24- and 21-day regimens of both drospirenone and norethisterone, respectively, showed also lower contraceptive failure rates for 24-day regimens.

In 8252 woman-years of adolescent COC exposure, 228 unintended pregnancies occurred in users of preparations containing $20 \mu \mathrm{g}$ ethinylestradiol. Based on strict criteria - intake in accordance with package insert - only $7 \%$ of the unintended adolescent pregnancies occurred during fully compliant use. This is substantially lower than the already very low number in adult women (14\%).
The PI for adolescents using 21and 24-day regimens was 5.1 (95\% CI 3.7-6.8) and 2.5 (95\% CI 2.1-2.9), respectively. In users of 21 -day regimens, the PI was $75 \%$ higher in adolescents compared to adults, while in users of 24-day regimens the PI in adolescents was only $34 \%$ higher. The adjusted HR for all 24- versus 21-day regimens in adolescents was 0.5 (95\% CI $0.4-0.7$ ). If the comparison is restricted to only COCs with a 24-day regimen that contain a progestogen with a long half-life (in our study a 24-day regime of $3 \mathrm{mg}$ drospirenone and $20 \mu \mathrm{g}$ ethinylestradiol) versus all 21-day regimens with $20 \mu \mathrm{g}$ ethinylestradiol the adjusted $\mathrm{HR}$ is 0.4 (95\% CI 0.3-0.5). A direct comparison of adolescent users of 24-day regimens containing drospirenone and norethisterone acetate, respectively, showed that using a progestogen with a long half-life has the potential to improve contraceptive effectiveness even further (adjusted HR 0.5, 95\% CI 0.4-0.7).

Overall, our subanalyses confirm that shortening the pill-free interval and using progestogens with a long halflife improve contraceptive effectiveness in adolescent COC users. This effect is even more pronounced compared to the improvement seen in adult users.

Juergen Dinger, MD, PhD

ZEG - Berlin Center for Epidemiology and Health Research, Berlin, Germany; dinger@zeg-berlin.de

Competing interests The study was supported with an unrestricted grant from Bayer Schering Pharma, Germany.

J Fam Plann Reprod Health Care 2011;37:118. doi:10.1136/jprhc.2011.0092

\section{REFERENCE}

1 Dinger J, Minh TD, Buttmann N, et al. Effectiveness of oral contraceptive pills in a large U.S. cohort comparing progestogen and regimen. Obstet Gynecol 2011;117:33-40. 